\title{
Pembrolizumab-Induced Lichen Planus on the Scalp of a Patient with Non- Small-Cell Lung Carcinoma
}

\author{
Aya Yamashita Eijiro Akasaka Hajime Nakano Daisuke Sawamura \\ Department of Dermatology, Hirosaki University Graduate School of Medicine, Hirosaki, Japan
}

\author{
Keywords \\ Pembrolizumab · Immune-related adverse effect $\cdot$ Immune checkpoint inhibitor $\cdot$ Lichen \\ planus $\cdot$ Scalp
}

\begin{abstract}
A 67-year-old man with non-small-cell lung carcinoma was referred to our department because of a pruritic rash on his head and upper extremities. Prior to the development of the rash, he had received 4 cycles of combination therapy with pemetrexed, carboplatin, and pembrolizumab, followed by 2 cycles of pembrolizumab monotherapy. On physical examination, violaceous scaly erythema grouped on his scalp and upper extremities. Histologically, the scalp lesions demonstrated irregular acanthosis that formed a characteristic saw-tooth appearance with hypergranulosis and typical lichenoid tissue reaction. These findings suggested that the scalp lesions were lichen planus. Two-week administration of topical corticosteroid dramatically improved the rash. Immunotherapy with pembrolizumab, an anti-PD-1 antibody, can induce T-cell activation that results in various immune-related adverse effects such as lichenoid tissue reaction. However, lichen planus is generally found on the extremities and/or oral mucosa, and unlike in this case, the scalp is rarely affected. Although the exact mechanism underlying predominant scalp involvement is unknown, the present case indicates that anti-PD-1 therapy-induced lichen planus can develop not only on the extremities and oral mucosa but also on the scalp. Interestingly, the lesions were not induced by the combination of chemotherapy and pembrolizumab; rather, they occurred soon after initiation of pembrolizumab monotherapy. In the present case, pembrolizumab-induced T-cell activation which triggered lichenoid tissue reaction may have been suppressed by chemotherapy-induced immunosuppression. Dermatologists should have a thorough knowledge of the cutaneous lesions that manifest as irAEs of anti-PD-1 therapy.
\end{abstract}




\section{Introduction}

Pembrolizumab is a humanized antibody to programmed death receptor 1 (PD-1) that inhibits the interaction between PD-1 and its ligand, programmed death ligand 1 (PD-L1). While immunotherapies with anti-PD-1 antibodies have shown significant clinical efficacy in the treatment of advanced-stage melanoma, non-small-cell lung carcinoma, and several other malignant diseases, they can induce T-cell activation that causes various immune-related adverse effects (irAEs) including maculopapular rash, vitiligo, pruritis, thyroiditis, hepatitis, diabetes mellitus, hypophysitis, and myasthenia gravis [1-3]. In addition, recent studies reported that lichen planus (LP) is also a common cutaneous irAE associated with anti-PD-1 therapies [2-6]. LP is a T-cell-mediated inflammatory keratinizing disease that develops in skin and mucosa, and LP involving the scalp has rarely been described [7]. We herein present an unusual case of pembrolizumab-induced LP that occurred on the scalp of a patient with lung adenocarcinoma.

\section{Case Report/Case Presentation}

A 67-year-old man with non-small-cell lung carcinoma was referred to our department because of 1-month history of a pruritic rash on his head and upper extremities. Prior to the development of the rash, he had received 4 cycles of combination therapy with pemetrexed, carboplatin, and pembrolizumab, followed by 2 cycles of pembrolizumab monotherapy. On physical examination, violaceous, scaly, erythematous plaques with a diameter of 1-2 cm were grouped on the patient's scalp (Fig. 1A). The plaques were surrounded by erythema and pigmentation. Similar keratotic erythematous lesions were scattered on the dorsum of the patient's hands and forearms (Fig. 1B). Dermoscopic examination of a scalp lesion demonstrated a whitish area at the center of the lesion, surrounded by dotted or linear hyperpigmentation (Fig. 1C). A skin biopsy specimen obtained from the scalp lesion showed orthokeratotic hyperkeratosis, irregular acanthosis that formed a characteristic saw-tooth appearance with hypergranulosis, and a lichenoid reaction characterized by liquefaction degeneration with prominent necrosis of individual keratinocytes and bandlike lymphocytic infiltrates (Fig. 1D, E). Two-week administration of topical corticosteroids dramatically improved the rash.

\section{Discussion/Conclusion}

In the present case, erythematous plaques developed mainly on the patient's scalp. Generally, LP lesions occur preferentially on the extremities and/or oral mucosa, while the scalp is rarely affected. LP-like lesions accompanied by scarring alopecia, a syndrome referred to as lichen planopilaris, was reported in 1 patient treated with pembrolizumab [7]. The patient in the present case showed no fibrosing alopecia, and band-like lymphocytic infiltration had mainly an interfollicular rather than folliculocentric distribution. In addition, histopathological examination revealed typical histological features of LP, such as band-like lymphocytic infiltration in the superficial dermis, liquefaction degeneration, and individual cell necrosis of the basal layer. These findings suggest that the scalp lesions were LP rather than lichen planopilaris. Although the exact mechanism underlying predominant scalp involvement is unknown, the present case indicates that anti-PD-1 therapy-induced LP can develop not only on the extremities and oral mucosa but also on the scalp.

\section{Karger'}


Case Reports

in Dermatology

\begin{tabular}{l|l}
\hline Case Rep Dermatol 2021;13:487-491 \\
\hline DOI: 10.1159/000519486 & $\begin{array}{l}\text { @ } 2021 \text { The Author(s). Published by S. Karger AG, Basel } \\
\text { www.karger.com/cde }\end{array}$ \\
\hline
\end{tabular}

Yamashita et al.: Pembrolizumab-Induced Lichen Planus on the Scalp
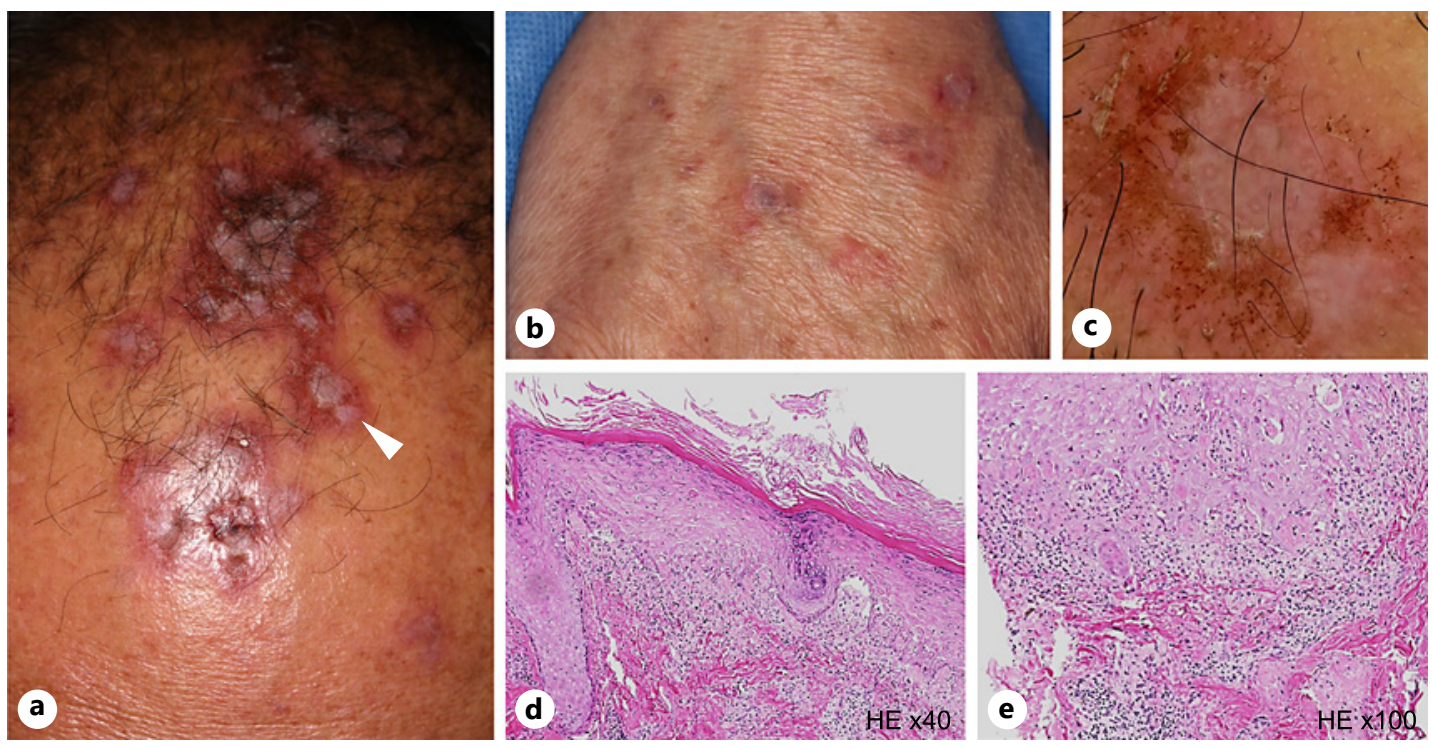

Fig. 1. Clinical features of lichen planus in the context of pembrolizumab therapy. A Violaceous, scaly, erythematous plaques with a diameter of 1-2 cm were grouped on the patient's scalp. Dermoscopic findings of the lesion indicated by the arrowhead are presented in (C). B Several violaceous, scaly, erythematous lesions are distributed on the dorsum of the hands. $\mathbf{C}$ Dermoscopic findings of the scalp lesion in the context of pembrolizumab therapy (arrowhead in A). There is a whitish structure at the center of the lesion accompanied by peripheral pigmentation. D, E Histopathology of lichen planus on the scalp in the context of pembrolizumab therapy. D At low-power view, hyperkeratosis, irregular acanthosis with hypergranulosis, liquefaction degeneration, and necrosis of individual keratinocytes were observed. E Band-like lymphocytic infiltrates at the dermal-epidermal junction are noted. Both (D, E) are hematoxylin and eosin stain. Original magnifications: $\times 40$ (D) and $\times 100$ (E).

The histological features described above also suggest that activated $\mathrm{T}$ lymphocytes attack basal keratinocytes, including epidermal stem cells. Damaged basal keratinocytes have decreased proliferation potential, causing them to exhibit delayed upward movement (in other words, an extended epidermal turnover time), followed by hypergranulosis and retention hyperkeratosis. Interestingly, the stratum corneum shows compact hyperkeratosis accompanied by cracks parallel to the skin surface without a basket-weave like structure. Oyama et al. [8] reported that in the normal stratum corneum, desmoglein 1 was distributed only in the lateral cell-cell contact areas of the plasma membrane and not on the ventral plasma membrane, while in LP, desmoglein 1 was observed homogenously on the ventral surface of corneocytes. This homogenous distribution of desmoglein 1 may contribute to loss of flexibility and to compact hyperkeratosis with cracks, which together with hypergranulosis result in the scaly, whitish, smooth surface of the lesions on clinical and dermoscopic examination.

Although the pathogenesis of the lichenoid reaction as a cutaneous irAE remains to be elucidated, several mechanisms have been proposed; for instance, the reaction may be mediated by an unmasked antigen, it may be generated by generalized upregulation of the immune system, and/or a neoantigen may be directly created by the anti-PD-1 antibody [9]. In any case, histopathological findings indicate a T-cell-mediated autoimmune process. Interestingly, in the present case, the LP lesions were not induced by the combination of chemotherapy and pembrolizumab; rather, they occurred soon after initiation of pembrolizumab monotherapy. Maarouf et al. [10] also reported a similar case of nivolumab-induced LP that had previously improved after chemotherapy for non-small-cell 
lung cancer. Anti-PD-1 therapy interrupts the PD-1/PD-L1 interaction and thereby activates $T$ cells, which may trigger an autoimmune response against keratinocytes and finally induce LP. Although it is difficult to conclude that delayed development of LP in our case is related to chemotherapy as irAEs sometimes occur long term after initiation of antiPD-1 therapies even without chemotherapy, we hypothesize that chemotherapy-induced immunosuppression may have attenuated T-cell activity and then suppressed development of LP lesions.

Basically, anti-PD-1 therapy should not be terminated due to skin rash since most of previously reported cases with LP induced by anti-PD-1 antibodies were successfully treated with topical corticosteroid and did not require cessation of anti-PD-1 antibodies. However, some cases show severe clinical manifestation in which LP lesions occurred all over their body or intractable oral ulcers. In such cases, termination of anti-PD-1 therapy and high dose of systemic corticosteroid would be needed to improve the mucocutaneous lesions $[1,4,7,9]$.

In conclusion, we demonstrated an unusual case of LP as an irAE of pembrolizumab that developed preferentially on the scalp. Dermatologists should have a thorough knowledge of the cutaneous lesions that manifest as irAEs of anti-PD-1 therapy.

\section{Statement of Ethics}

Written informed consent was obtained from the patient for publication of this case report and images. The study is exempt from ethics committee approval because all data in this study were obtained not for research but for diagnosis of the patient. Moreover, the research was conducted ethically in accordance with the World Medical Association Declaration of Helsinki.

\section{Conflict of Interest Statement}

The authors have no conflicts of interest to declare.

\section{Funding Sources}

The authors are not being funded.

\section{Author Contributions}

Eijiro Akasaka was involved in direct management of the patient. Aya Yamashita drafted the first manuscript and reviewed the literature. Hajime Nakano and Daisuke Sawamura supervised the manuscript drafting. All authors read and approved the final manuscript.

\section{Data Availability Statement}

All data generated or analyzed during this study are included in this article. Further enquiries can be directed to the corresponding author. 


\section{References}

1 Hofmann L, Forschner A, Loquai C, Goldinger SM, Zimmer L, Ugurel S, et al. Cutaneous, gastrointestinal, hepatic, endocrine, and renal side-effects of anti-PD-1 therapy. Eur J Cancer. 2016;60:190-209.

2 Belum VR, Benhuri B, Postow MA, Hellmann MD, Lesokhin AM, Segal NH, et al. Characterisation and management of dermatologic adverse events to agents targeting the PD-1 receptor. Eur J Cancer. 2016;60:12-25.

3 Hwang SJ, Carlos G, Wakade D, Byth K, Kong BY, Chou S, et al. Cutaneous adverse events (AEs) of antiprogrammed cell death (PD)-1 therapy in patients with metastatic melanoma: a single-institution cohort. J Am Acad Dermatol. 2016 Mar; 74(3):455-e1.

4 Denny J, Chong H, Akhras V. Lichen planus in a patient treated with pembrolizumab for metastatic malignant melanoma. Clin Exp Dermatol. 2018 Apr;43(3):354-6.

5 Komori T, Honda T, Irie H, Otsuka A, Kabashima K. Lichen planus in irradiated skin during nivolumab treatment. Acta Derm Venereol. 2017 10;97(3):391-2.

6 Yilmaz M, Mese SG, Celik U. Nivolumab-induced lichen planus. J Oncol Pharm Pract. 2020 Apr;26(3):758-60.

7 Cogen AL, Parekh V, Gangadhar T, Lipoff JB. Lichen planopilaris associated with pembrolizumab in a patient with metastatic melanoma. JAAD Case Rep. 2018 Jan;4(2):132-4.

8 Oyama Z, Naoe Y, Kimura H, Masunaga T, Seishima M, Aoyama Y, et al. New non-invasive method for evaluation of the stratum corneum structure in diseases with abnormal keratinization by immunofluorescence microscopy of desmoglein 1 distribution in tape-stripped samples. J Dermatol. 2010 Oct;37(10):873-81.

9 Shi VJ, Rodic N, Gettinger S, Leventhal JS, Neckman JP, Girardi M, et al. Clinical and histologic features of lichenoid mucocutaneous eruptions due to anti-programmed cell death 1 and anti-programmed cell death ligand 1 immunotherapy. JAMA Dermatol. 2016;152(10):1128-36.

10 Maarouf M, Alexander C, Shi VY. Nivolumab reactivation of hypertrophic lichen planus, a case report and review of published literature. Dermatol Online J. 2018 Jan;24(1):13030/qt4xf465w6. 